\title{
Clima emocional: El efecto mediacional de medidas de justicia restaurativa y del compartir social
}

\author{
Juan Bombelli¹ ${ }^{1}$ Marcela Muratori² y Elena Zubieta ${ }^{3}$
}

\begin{abstract}
RESUMEN
Se realizó un estudio empírico con 470 personas $\left(40.2 \%\right.$ hombres, $59.8 \%$ mujeres, $M_{\mathrm{EDAD}}=$ 35.26 años, $D E=13.29$ ). El objetivo fue explorar la percepción del clima social emocional en una muestra de personas con diferentes niveles de afectación por la violencia política durante la última dictadura militar argentina e indagar en algunas variables mediadoras de dicha percepción. Se halló una considerable aprobación al trabajo de la CONADEP y de los Juicios a las Juntas, una pobre percepción de sinceridad a las disculpas públicas efectuadas por representantes de instituciones, y bajo compartir social sobre lo sucedido en el período en cuestión. Además, se corroboró que el nivel de victimización predice el clima emocional positivo, y más aún cuando se introducen como mediadores el compartir social, la aprobación de la CONADEP y de los Juicios (con leve efecto), y la sinceridad atribuida a las disculpas.
\end{abstract}

Palabras Clave: clima emocional, justicia restaurativa, compartir social, dictadura militar.

\section{Emotional climate: The mediational effect of measures of restorative justice and social sharing}

\begin{abstract}
This paper, in which 470 people with different levels of victimization by dictatorship's violence participated, explores the perception of the socioemotional climate and tends to identify the meditational effect of some variables. Results show important levels of approvement to the work of the Argentinean Truth Commission and to the Trials to the military Juntas, low sincerity perceived in apologies made by institutional representants, and minimal social sharing about the past violence. Victimization predicts positive emotional climate, but with higher effect when social sharing, approvement of Truth Commission's work and Junta's Trials, and apologies' sincerity are mediating.

Keywords: emotional climate, transitional justice, social sharing, military dictatorship.
\end{abstract}

Durante el período de 1976-1983, Argentina estuvo sometida a una dictadura cívico militar donde la censura, la violencia y el miedo se instalaron mediante el ejercicio del poder de las Juntas de Gobierno Militares. Este último golpe de Estado, orquestado por el Teniente General Jorge Rafael Videla; Almirante Emilio Massera y Brigadier General Orlando Agosti, fue el

\footnotetext{
1 Universidad Católica de La Plata, Argentina.

2 Consejo Nacional de Investigaciones Científicas y Técnicas (CONICET), Universidad de Buenos Aires, Universidad Católica Argentina, Argentina.

${ }^{3}$ Consejo Nacional de Investigaciones Científicas y Técnicas (CONICET), Universidad de Buenos Aires, Argentina; elenazubieta@hotmail.com
} 
culmen de una larga lista de golpes de Estado en Argentina que, por otra parte, estaban en sintonía con un fenómeno de época latinoamericano. Durante la década del 70 se produjeron los golpes de Estado en Bolivia, en 1971; en Chile y Uruguay, en 1973; en Argentina, en 1976. Pueden considerarse también las dictaduras en Paraguay desde el año 1954, y en Brasil desde 1964 (Victoriano Serrano, 2010). Sin embargo, es en esta década del '70 donde las dictaduras exhiben su versión más represiva y sangrienta, y en Argentina esto se da de un modo particular, en especial por la apelación sostenida a la desaparición forzada de personas como forma de control social (Calcagno, 2013; Instituto Espacio para la Memoria, 2013). En su análisis sobre el caso local, Robben (2005) afirma que, sin lugar a duda, debido a la violencia política, la impunidad con la que se actuó desde el Estado Nacional tomado por los militares y la extensiva utilización de la represión como modo sistemático de regulación social, se puede considerar al último período de gobierno militar como un trauma social para la nación Argentina. A propósito, Martín-Baró (2003) entiende que dichos traumas provocan efectos psicosociales, más allá de las heridas físicas o emocionales que puedan acarrear en individuos concretos. En términos de la dimensión emocional del trauma, una de las consecuencias psicosociales de la violencia sociopolítica radica en una determinada percepción del clima emocional, entendiendo por este a lo que de Rivera (1992) define como la predominancia y saliencia relativa de un conjunto de escenarios emocionales. En otras palabras, sería aquel conjunto de reacciones e interacciones sociales, cargadas afectivamente, que predominan durante un periodo sociopolitico y que impregnan las relaciones interpersonales. Páez et al. (1996) amplían la definición cuando indican que se trata de un estado de ánimo colectivo caracterizado por:

a) la prevalencia de ciertas emociones vivenciadas por los miembros de un grupo, tales como la alegría o el miedo y la tristeza;

b) la apreciación por parte de las personas de un determinado ambiente social;

c) el predominio de un conjunto de creencias compartidas sobre el entorno social, las instituciones (confianza versus desconfianza), el mundo en general y el futuro social (optimista y de esperanza, en oposición a la desesperanza);

d) la existencia de ciertas disposiciones a actuar asociadas a las funciones sociales de las emociones.

Un clima socioemocional positivo influye sobre todo en los aspectos de aceptación de los otros y en percibir un progreso social positivo, mientras que un clima negativo los afecta negativamente con similar intensidad. Un clima positivo refuerza en mayor medida la integración social y el carácter comprensible y controlable del medio de lo que lo debilita un clima negativo. Finalmente, la relación es débil, aunque significativa para el clima positivo con la percepción de que se aporta algo útil a la colectividad o contribución social (Bilbao et. al, 2011).

Así como la violencia colectiva construye un determinado clima social emocional, el afrontamiento activo y colectivo como rituales de justicia, y el compartir social de las emociones, puede influirlo. Y esta influencia es probable que varie, que haya una percepción diferencial en función del grado de afectación: las víctimas tendrían que percibirlo más negativo, pero, si hay justicia y mecanismos de afrontamiento colectivo, la situación debería revertirse (Techio et al., 2011). 
Los climas emocionales también están claramente condicionados por hechos objetivos, cambios institucionales y politicas públicas que crean experiencias compartidas, así por cómo la gente común se comporta. Este condicionamiento por el comportamiento común o cotidiano hace del clima emocional no solo un proceso macrosocial sino también microsocial. Rimé (2005) sostiene que es generado por la necesidad de la gente de hablar con otros de sus experiencias emocionales y es este compartir social el que refuerza las emociones y ayuda a construir convergencia y semejanza en las emociones percibidas.

Los rituales de justicia, como las Comisiones de Verdad (en adelante CV), también aumentan la percepción de hostilidad y conflicto intergrupal. El afrontamiento activo contra la injusticia se asocia a la reactivación de emociones negativas aun cuando el apoyo social y las creencias positivas se refuerzan. Son los beneficios colectivos de la participación en formas de afrontamiento, como ser parte de comisiones de verdad, el castigo simbólico a los perpetradores o el reconocimiento moral a la dignidad de las víctimas, los que llevan a que los eventos sean útiles herramientas sociales para combatir climas emocionales negativos y construir culturas de paz (Zubieta, Bombelli, \& Muratori, 2016).

De lo anterior se deduce la relevancia de la percepción de un determinado clima emocional, toda vez que colabora en crear realidades subjetivas que rigen la conducta, establecen pautas sobre la expresividad emocional y posibilitan un marco de continuidad o intermitencia en los vínculos interpersonales que tienen un rol fundamental en el mantenimiento de la salud física y mental de los individuos, tanto como su participación en el entorno social (Blanco \& Díaz, 2004; Zubieta, Delfino, \& Fernández, 2008). A partir de las ideas de Martín-Baró (2003), como producto del terror sociopolítico las personas afectadas quedan con una vida social estrecha y rígida, se socavan sus relaciones interpersonales y se deteriora su convivencia social. Para Lira y Castillo (1991), cuando miles de personas son amenazadas por un régimen político, la sensación de vulnerabilidad y de miedo incide sobre la conducta y la conciencia del grupo humano afectado.

Entonces: ¿Qué se puede hacer para restablecer ciertas condiciones de convivencia social que restauren un orden de cosas dinamitado por el terror sociopolítico? ¿Es acaso esto posible? En respuesta a la primera pregunta, es necesario considerar la aplicación de un conjunto de medidas que se establecen en el marco de la Justicia Transicional. Es decir: aquel modelo de justicia que se administra luego de violaciones severas a los derechos humanos por parte de agentes estatales y/o fuerzas armadas de oposición. Dentro de ese conjunto de medidas se pueden identificar la creación y el desempeño de las CV que, luego de períodos de violencia colectiva extrema, buscan construir una historia alternativa respecto de aquellos hechos lamentables ya perpetuados, de modo que las condiciones sociales que habian dado lugar a semejantes hechos fueran suplidas por otras que no permitieran ciclos de venganza o el resurgimiento de la violencia (Teitel, 2003). Una CV es un organismo de carácter temporal instituido para investigar las violaciones a los derechos humanos realizadas en un país (Hayner, 1994). Estos organismos colaboran aportando información que luego puede o no ser utilizada por las instituciones responsables de implementar la Justicia (Avruch, 2010). Además, las CV son un foro en el que las víctimas pueden entregar su testimonio, hablar sobre aquello que les ocurrió en el pasado y transmitir su 
experiencia, validando su sufrimiento y promoviendo la empatía entre quienes los escuchan (Beristain, 2005). Además de estas CV, para restablecer el estado democrático de gobierno pueden aplicarse medidas de justicia retributiva (juicios a los responsables), o restaurativa, mediante rituales de recuerdo y conmemoraciones, pedidos de disculpas públicos, resarcimientos económicos a las víctimas, etc. (Andrieu, 2010). Todas estas medidas pueden ser entendidas como rituales transicionales que buscan vehiculizar un conjunto de mecanismos que, sin dar cabida a la impunidad, luchan por la consolidación de normas y el refuerzo de la cohesión social en pos de evitar el resurgimiento de la violencia, recuperar la confianza interpersonal e institucional vulnerada en el período de violencia y, así, garantizar la paz y la convivencia armónica (Beristain, Páez, Rimé, \& Kanyangara, 2010; Teitel, 2003; Wilson, 1995).

Diversos estudios señalan los beneficios salutogénicos del compartir social de las experiencias emocionales, sobre todo cuando estas son intensas (Frattaroli, 2006; Pennebaker, Zech, \& Rimé, 2001; Weinman, Ebrecht, Scott, Walburn, \& Dyson, 2008), o el impacto negativo sobre el bienestar subjetivo de la inhibición de la expresión emocional (Finkenauer \& Rimé, 1998). Otros estudios que abordan la temática del impacto psicosocial de la violencia colectiva revelan que las víctimas directas y las generaciones más jóvenes son quienes más necesidad sintieron de hablar sobre lo sucedido en el pasado (Arnoso, Cárdenas y Páez, 2012), aunque en una investigación realizada con población argentina se observa que tanto quienes vivieron la dictadura como quienes nacieron en democracia muestran una alta necesidad de hablar sobre el pasado violento (Arnoso, Cárdenas, Muratori, Zubieta, Páez y Bombelli, 2014). Asimismo, en un trabajo transcultural que incluyó 530 sujetos de seis países distintos, se detecta que el mayor recuerdo de eventos traumáticos se asocia a un mayor clima emocional negativo percibido y, en esa tendencia, un menor clima emocional positivo, y que mientras más se habla sobre aquellos, menor es el clima positivo percibido y mayor el clima negativo (Páez, Ruiz, Gailly, Kornblit, Wiesenfeld y Vidal, 1996).

En este marco, se realizó un estudio empírico con el objetivo de explorar la percepción del clima social emocional en una muestra de personas con diferentes niveles de afectación por la violencia política durante la última dictadura militar argentina. Se evaluó también en qué medida el compartir social, la aprobación del trabajo de la CONADEP y de los Juicios a las Juntas, y la sinceridad atribuida a las disculpas, actúan como factores mediadores en la percepción de un clima emocional positivo o negativo.

\section{MÉTODO}

Diseño. Estudio descriptivo, de diseño no experimental transversal, con población general de distintas ciudades de Argentina.

Muestra. No probabilística intencional, está compuesta por 470 participantes de los cuales el $40.2 \%(n=189)$ son hombres y el 59.8\% $(n=281)$ mujeres. La edad oscila entre los 18 y los 83 años, con una edad media de 35.26 años $(D E=13.29)$. Respecto al posicionamiento ideológico, la mitad se define con una orientación política de centro $(n=218 ; 49 \%)$, un $30.6 \%(n=$ 136) se identifica con la izquierda y un $20.4 \%(n=91)$ se ubica a la derecha. Del total de la muestra, el $6.6 \%(n=31)$ afirma ser victima directa de la 
dictadura, el $20.6 \%(n=97)$ víctima indirecta, y el $65.5 \%(n=308)$ se define como no afectado.

Instrumento. En el encabezado del cuestionario figuraba el Consentimiento Informado que todo participante debía completar antes de iniciar la tarea solicitada, y solo una vez firmado se proseguía. Los participantes, luego, respondieron un cuestionario de formato autoadministrado integrado por preguntas sobre:

Nivel de exposición a la violencia en tres niveles: víctimas directas (personas que fueron víctimas de la violencia) -en adelante VD-; víctimas indirectas (personas que tienen víctimas entre sus familiares y amigos más cercanos) -en adelante VI- y población no afectada -en adelante NA-.

Compartir social sobre el período de dictadura, violencia, la actuación de la CONADEP y el Informe Nunca Más. Con una lista de seis items, que se responden en un continuo de 1 (Nada) a 4 (Mucho), se evalúa la necesidad y frecuencia de hablar de esos temas, así como la inhibición de referirse a los mismos ("¿Ha hablado acerca de la CONADEP y el Informe Nunca Más con otras personas después de su publicación?; ¿Ha hablado de la época de la dictadura con otras personas después de la entrega del Informe Nunca Más?; ¿Ha hablado del Informe Nunca Más con otras personas este mes?; ¿Ha sentido necesidad de hablar de la dictadura y los hechos que ocurrieron en el periodo 1970-1983?; ¿Existen algunos aspectos de la violencia de aquella época de los cuales nunca habló con nadie?; ¿Existen algunos aspectos de la violencia de la época de los cuales no quiere hablar en absoluto?”).

Una pregunta dirigida a medir el grado de aprobación del trabajo realizado por la CONADEP ("Con respecto a la actuación de la CONADEP, diría usted que..."), con una opción de respuesta que va de 1 (lo desaprueba totalmente) a 4 (lo aprueba totalmente).

Una pregunta dirigida a medir el grado de aprobación del Juicio a las Juntas Militares ("Con respecto a los Juicios a las Juntas Militares, diría usted que..."), con una opción de respuesta que va de 1 (lo desaprueba totalmente) a 4 (lo aprueba totalmente).

Grado de sinceridad de los pedidos de disculpas de Néstor Kirchner y Martín Balza; 1 (Nada) a 4 (Mucho).

Una versión abreviada de la escala de clima emocional del país (Páez et al., 1997). En cuatro ítems con un continuo de respuesta donde 1 (nada) y 5 (mucho), evalúa en qué medida la percepción del clima social del país es de solidaridad y ayuda mutua, de confianza en las instituciones, de enojo y hostilidad entre las personas y grupos, así como un bajo estado de ánimo caracterizado por la tristeza y la pasividad.

\section{RESULTADOS}

Los resultados se exponen con la siguiente secuencia. En primer lugar, se reportan los resultados descriptivos de las variables analizadas. En segundo término, se analizan las diferencias en función de la variable victimización. Por último, se muestra lo obtenido en los análisis mediacionales realizados con el propósito de evaluar la medida en que el compartir social, la aprobación del trabajo de la CONADEP y de los Juicios a las Juntas, y la sinceridad atribuida a las disculpas, intervienen en la percepción de un clima social emocional positivo o negativo. 
Existe un amplio consenso en la valoración muy positiva del trabajo realizado por la CONADEP $(M=3.46, D E=.68)$. Al respecto, se encuentran diferencias significativas al considerar el nivel de victimización $F(5,526), p=$ $.004, \mathrm{VD}=3.56, \mathrm{VI}=3.64, \mathrm{NA}=3.39$. Las víctimas directas e indirectas se posicionan como un grupo homogéneo valorando más el trabajo de la $\mathrm{CV}$, en comparación con lo que estiman los no afectados por la violencia.

Respecto de la aprobación de los Juicios a las Juntas del Gobierno Militar, se observan puntajes medios muy elevados $(M=3.59, D E=.71)$, más elevados aún que los reportados en relación con la CONADEP. Surgen también aquí diferencias en función del grado de victimización de las personas, $F(4,967), p$ $=.007, \mathrm{VD}=3.51, \mathrm{VI}=3.79, \mathrm{NA}=3.54$, siendo las víctimas indirectas quienes más aprueban los Juicios llevados a cabo en 1985. La prueba post-hoc Scheffé revela que las víctimas indirectas se destacan por una mayor aprobación de los juicios por sobre los no afectados y las víctimas directas, que funcionan como un subgrupo homogéneo.

A nivel general, se detecta una baja necesidad general de hablar sobre lo sucedido $(M=1.92, D E=.68)$, donde las víctimas directas e indirectas, en comparación con los no afectados por la violencia, muestran una mayor necesidad de hablar sobre lo sucedido, $F(31,986), p<.001, \mathrm{VD}=2.42$, $\mathrm{VI}=$ 2.22, $\mathrm{NA}=1.71$.

Al evaluar la sinceridad atribuida a las disculpas efectuadas por el presidente Néstor Kirchner y el ex Jefe del Ejército Martín Balza, los datos indican que no se las consideran muy sinceras $(M=2.20, D E=.80)$ aunque surgen diferencias significativas en función del grado de victimización, $F(4,976), p=.007, \mathrm{VD}=2.44, \mathrm{VI}=2.37, \mathrm{NA}=2.12$. Son las víctimas directas quienes más sinceras consideran las disculpas. Además, las pruebas post-hoc Scheffé indican que los no afectados se diferencian de los participantes que sufrieron algún tipo de victimización, atribuyendo menor sinceridad a las disculpas en cuestión.

Por último, se observa una mayor percepción general de clima emocional negativo $(M=3.27, D E=.99)$ por sobre el positivo $(M=2.57, D E=.86)$, detectándose diferencias significativas según el grado de victimización, $F(5$, $381), p=.005, \mathrm{VD}=3.15, \mathrm{VI}=3.00, \mathrm{NA}=3.36$, en las que los no afectados por la violencia son quienes más perciben que en el entorno y entre las personas prevalecen emociones de tristeza y enojo. En lo que respecta al clima positivo, las diferencias significativas se observan particularmente entre las víctimas directas y los no afectados donde los últimos son quienes menos consideran que haya solidaridad, ayuda mutua y confianza en las instituciones, $F(6,201), p=.002, \mathrm{VD}=2.89, \mathrm{VI}=2.72, \mathrm{NA}=2.48$.

\section{Análisis Mediacionales: diferencias según el grado de victimización en clima emocional con distintas variables como mediadoras}

Para cotejar la hipótesis de efecto indirecto del grado de victimización sobre el clima emocional a través de las variables analizadas, se realizó un análisis mediacional. Con el objetivo de contrastar la hipótesis de mediación se utilizó el macro de SPSS con el método de bootstrap para efectos indirectos, el cual estima el efecto indirecto, los errores estándar y los intervalos de confianza derivados de la distribución bootstrap (Preacher \& Hayes, 2008). El efecto indirecto resulta significativo si el intervalo de confianza no pasa por el valor de cero. Asimismo, se propone que el efecto total no necesariamente 
tiene que ser significativo para que haya mediación, ya que lo importante es la diferencia absoluta entre el efecto total y el indirecto (Preacher \& Hayes, 2008).

Los resultados obtenidos indican, en primer lugar, que el grado de afectación es un predictor significativo en la aprobación del trabajo realizado por la CV argentina, $B=.12, D E=0.04, t=2.61, p=.01$, IC 95\% [.032, .226], donde un mayor grado de victimización se asocia a una mayor aprobación. Además, esta aprobación se asocia significativamente con la percepción de un clima emocional positivo, $B=.19, D T=0.06, t=3.20, p=.00$, IC 95\% [.074, .309]. Finalmente, si bien el grado de victimización es un predictor significativo de clima emocional positivo, efecto total: $B=.21, D E=0.06, t=$ $3.38, p<.001$, IC 95\% [.089, .335], el aprobar la labor de la CONADEP tiene un rol mediador en esa relación, efecto directo: $B=.18, D E=0.06, t=2.99, p$ $=.00$, IC 95\% [.064, .310], efecto indirecto: $B=.02$, Boot $D E=0.01$, IC 95\% $[.004, .054]$.

Por otra parte, los análisis también sugieren que el grado de victimización es un predictor significativo en la aprobación del trabajo realizado por la CONADEP, $B=.13, D E=0.05, t=2.63, p=.01$, IC 95\% [.033, .228], toda vez que a mayor grado de victimización las personas evalúan mejor la labor de la comisión. Asimismo, la evaluación mencionada se asocia, aunque solo tendencialmente, a una percepción del clima emocional negativo $B=.04, D E=0.07, t=.56, p=.57$, IC 95\% [-.096, .174]. Por último, el grado de victimización, además de ser un predictor significativo en lo que respecta a la percepción de un clima negativo caracterizado por la creencia de que la mayoria de sus conciudadanos sienten enojo y tristeza, muestra un leve efecto mediador explicado por la aprobación de todo el trabajo realizado por la CV local, efecto total: $B=-.17, D E=0.07, t=-2.46, p=.01$, IC 95\% [-.316, -.036]; efecto directo: $B=-.18, D E=0.07, t=-2.51, p=.01$, IC 95\% [-.322, -.039]; efecto indirecto: $B=.01$, Boot $D E=0.01$, IC 95\% [-.012, .029].

Al incluir como variable mediadora la aprobación de los Juicios a las Juntas Militares, se observa que la victimización no es un indicador confiable de predicción de clima negativo, $B=.06, D E=0.05, t=1.15, p=.24$, IC 95\% [$.041, .161]$. Distinta es la capacidad predictiva de la aprobación de esos Juicios en la evaluación del clima emocional positivo, donde sí se observa una relación significativa, $B=.23, D T=0.06, t=4.12, p=.00$, IC 95\% [.119, .337]. Finalmente, se observa que la aprobación de los juicios penales a las Juntas de Comandantes de la Dictadura tiene un efecto mediador significativo entre la victimización y la percepción de clima emocional positivo, efecto total: $B=$ .23, $D E=0.06, t=3.72, p<.001$, IC 95\% [.108, .352]; efecto directo: $B=.22$, $D E=0.06, t=3.55, p<.001, \mathrm{IC} 95 \%[.097, .336]$; efecto indirecto $B=.01$, Boot $D E=0.01$, IC 95\% [-.010, .041].

Al considerar el efecto predictor de las variables del grado de victimización sobre la percepción del clima emocional negativo, y controlar el efecto mediador de la aprobación de los Juicios durante el gobierno de Raúl Alfonsín, se detecta que, al igual que en el análisis precedente, el grado de afectación por la violencia no predice la evaluación de los juicios, $B=.06, D E=$ $0.05, t=1.12, p=.26$, IC 95\% [-.043, .160]. Tampoco el grado de aprobación es un predictor de la percepción de un clima de enojo y tristeza $(B=.02, D E=$ $0.06, t=.26, p=.80$, IC 95\% [-.110, .144]. Sin embargo, se observa un infimo efecto mediador de la aprobación de los Juicios a las tres Juntas de Gobierno militar, entre la victimización y la percepción de clima emocional negativo, efecto total: $B=-.20, D E=0.07, t=-2.77, p=.01$, IC 95\% [-.336, -.057]; efecto 
directo: $B=-.20, D E=0.07, t=-2.77, p=.01$, IC 95\% [-.337, -.057]; efecto indirecto: $B=.00$, Boot $D E=0.01$, IC 95\% [-.006, .018].

Por su parte, el grado de victimización es un predictor significativo en la evaluación de la sinceridad de las disculpas ofrecidas por el presidente Néstor Kirchner y el ex Jefe del Ejército Martín Balza, $B=.18, D E=0.06, t=2.96, p=$ .00 , IC 95\% [.061, .395]. Además, la sinceridad atribuida a las disculpas también resulta un predictor significativo de la evaluación del clima emocional positivo, $B=.30, D E=0.05, t=5.93, p=.00$, IC 95\% [.200, .400]. Por último, la aprobación de las disculpas ofrecidas tiene un efecto mediador en la evaluación que los participantes hacen del clima emocional positivo, efecto total: $B=.21, D E=0.07, t=3.18, p=.00$, IC 95\% [.079, .337]; efecto directo: $B=.15, D E=0.06, t=2.41, p=.02$, IC 95\% [.028, .278]; efecto indirecto: $B$ $=.06, D E=0.06$, IC $95 \%[.015, .105]$.

En relación con el análisis previo, el grado de victimización resulta un predictor significativo en la evaluación de la sinceridad de las disculpas ofrecidas por los representantes del Gobierno Nacional y del Ejército, $B=.18$, $D E=0.06, t=2.91, p<.001$, IC 95\% [.058, .302]. Además, la sinceridad atribuida a esas disculpas también predice de forma inversa la evaluación del clima emocional negativo, $B=-.33, D E=0.06, t=-5.50, p<.001$, IC 95\% [$.444,-.210]$, al revelar que, a mayor sinceridad atribuida a las disculpas, menor es la percepción del clima emocional negativo. Por último, la aprobación de las disculpas ofrecidas no exhibe un efecto mediador en la percepción de los participantes de un clima emocional negativo, efecto total: $B$ $=-.17, D E=0.08, t=-2.28, p=.02$, IC 95\% [-.325, -.024]; efecto directo: $B=-$ $.11, D E=0.07, t=-1.55, p=.12$, IC 95\% [-.262, .031]).

Finalmente, el grado de victimización predice de manera significativa el compartir social, siendo que las personas más afectadas por la violencia son quienes manifestaron sentir una mayor necesidad de hablar sobre lo que les sucedió, $B=.36, D E=0.05, t=7.75, p<.001$, IC 95\% [.270, .454]. E1 compartir social también es un predictor significativo de la percepción de un clima emocional positivo, $B=.22, D E=0.06, t=3.61, p<.001$, IC 95\% [.099, .337], a la vez que exhibe un rol mediador en la evaluación del clima emocional positivo, efecto total: $B=.22, D E=0.06, t=3.66, p<.001$, IC $95 \%$ [.103, .343]; efecto directo: $B=.14, D E=0.06, t=2.25, p=.02$, IC 95\% [.018, .270]; efecto indirecto, $B=.08$, Boot $D E=0.03$, IC 95\% [.034, .135].

A su vez, quienes más compartieron sobre lo sucedido durante la dictadura fueron quienes evaluaban menos negativamente el clima emocional negativo, $B=-.21, D E=0.07, t=-2.94, p<.001$, IC 95\% [-.352, -.069], evidenciando los efectos positivos del compartir social de las emociones, aunque el compartir social no explica la capacidad predictiva de la victimización en la percepción del clima emocional negativo, efecto total: $B=-$ $.18, D E=0.07, t=-2.58, p=.01$, IC 95\% [-.325, -.044]; efecto directo: $B=-.11$, $D E=0.07, t=-1.46, p=.15$, IC 95\% [-.258, .038]).

\section{DISCUSIÓN Y COMENTARIOS}

El trabajo realizado aporta hallazgos interesantes respecto de la contribución de diversas medidas de Justicia Restaurativa. La extensa aprobación a las medidas evaluadas, como fueron los Juicios a los responsables de la violencia de Estado, y la Comisión de Verdad, encargada de aportar datos concretos y bien documentados del destino de los desaparecidos 
y de las circunstancias de su desaparición, son importantes medidas en la recuperación de un Estado de Derecho confiable, garante de paz y de una convivencia armoniosa necesaria para vivir en sociedad (Beristain et al., 2010; Teitel, 2003; Wilson, 1995). Aunque la ejecución de estas medidas sean herramientas útiles para paliar las consecuencias de la violencia, la poca sinceridad atribuida a las disculpas públicas ofrecidas por indiscutibles representantes del Poder Ejecutivo y de las Fuerzas Armadas, obstaculiza fuertemente el proceso de allanar el camino para lograr la empatía, la confianza, el perdón y la reconciliación intergrupal, desagraviar a las víctimas y restablecer la credibilidad institucional de las personas, tal como lo reportan estudios previos (Blatz, Schuman, \& Ross, 2012; Cehajic, Brown, \& Castano, 2008).

El grado de victimización resulta un aspecto de relevancia, ya que se observa que las personas que sufrieron de forma directa o indirecta la violencia durante la dictadura son quienes más aprobaron la labor de la CONADEP. Asimismo, las víctimas exhiben un importante grado de aprobación de los Juicios a las Juntas del Gobierno Militar, y son también las víctimas (considerándolas de forma amplia) quienes más necesidad sienten de compartir sus experiencias y sentimientos sobre lo que vivieron en aquel período histórico. Complementando lo anterior, los puntajes más elevados en quienes se consideraban víctimas directas respecto de la sinceridad de las disculpas pueden deberse a la mayor necesidad de dar sentido a lo sucedido, al ser estas disculpas una forma de reconocer su sufrimiento y comenzar a sanar las heridas de la violencia. Sin tales disculpas, o no creyéndolas sinceras, el mecanismo interno de las víctimas puede continuarse en un proceso rumiativo que aumente el resentimiento, la hostilidad y la desconfianza, todo lo cual afecta negativamente el bienestar, predisponiendo al aislamiento y al malestar social.

Los resultados relativos a una mayor percepción de clima negativo que positivo son coincidentes con los estudios previos que se vienen realizando hace algunos años en el ámbito local (Fernández, Mele, Bombelli, \& Zubieta, 2011; García Mazzieri, 2011; Muratori \& Zubieta, 2013; Zubieta, Delfino, \& Fernández, 2008), donde el clima predominantemente negativo se relaciona con la desconfianza en las instituciones y la percepción de problemas sociales como la inseguridad y la dificultad para encontrar trabajo o conseguir una vivienda propia. En este sentido, aun cuando pueda prevalecer la sensación de disconformidad con la situación actual percibida en la sociedad, para las víctimas -sean directas o indirectas- la temática de su experiencia durante la dictadura puede ofrecerse como un punto de referencia a partir del cual establecer evaluaciones sobre la actual situación colectiva. Desde esta hipótesis explicativa, entonces, los índices más elevados de clima emocional positivos tanto como los menores niveles de clima emocional negativo pueden deberse al efecto realmente restaurativo de las medidas implementadas, considerándolas herramientas eficaces en la reivindicación de derechos y un importante progreso democrático, aun cuando haya mucho más por hacer al respecto.

En relación con lo anterior, y fortaleciendo significativamente tal razonamiento, los análisis mediacionales dan cuenta de cómo las variables analizadas tienen un poder explicativo en cómo es percibido el clima emocional, sobre todo el clima emocional positivo de solidaridad, ayuda mutua y confianza en las instituciones. En líneas generales, el nivel de victimización 
fue un predictor significativo del clima emocional positivo, y explica mejor el modelo al ser moderado por el compartir social, la aprobación de la CONADEP y de los Juicios (con leve efecto mediador), y la sinceridad atribuida a las disculpas.

E1 otro análisis mediacional realizado arroja un dato valioso que guarda relación con cómo influyen el compartir social y la atribución de sinceridad de las disculpas públicas al momento de explicar una relación inversa entre la victimización y la percepción de clima emocional negativo.

Por último, la baja necesidad de hablar sobre lo sucedido podría explicarse como resultado de una cierta represión ejercida socialmente ante hechos traumáticos como fue la dictadura argentina, lo cual es una forma factible de afrontamiento. Los resultados de este estudio discrepan respecto de lo que encontraron Arnoso, Cárdenas, Páez y Beristain (2014) en su estudio sobre el impacto psicosocial de la Comisión de Verdad en Paraguay, donde los participantes mostraron una mayor necesidad de hablar sobre lo sucedido, indistintamente de su grado de afectación por la violencia.

En lo que hace a limitaciones del estudio, es preciso destacar que la naturaleza no probabilística de la muestra acota la interpretación de los resultados, así como su generalización. El diseño transversal es otra restricción importante que condiciona la causalidad de las conclusiones. Los trabajos realizados en el marco del proyecto transcultural en el que se inscribe la investigación se han orientado a la replicación en diferentes países del cono sur, así como a la profundización de diferentes aspectos relacionados con el impacto psicosocial de las medidas de justicia transicional.

Los análisis que anteceden a este trabajo han corroborado la incidencia de aspectos como la victimización o la afectación por la violencia tanto en el caso argentino (Arnoso, Muratori, Zubieta, Cárdenas, Bombelli, \& Páez, 2017; Bombelli, Muratori, Arnoso, Mele \& Zubieta, 2013; Zubieta, Bombelli \& Muratori, 2015; Zubieta, Bombelli \& Muratori, 2015), como chileno (Cárdenas, Páez, Arnoso, \& Rimé, 2013; Cárdenas, Páez, \& Rimé, 2013) y paraguayo (Arnoso, Muratori, Páez, Zubieta, \& Cárdenas, 2014). También se han estudiado los múltiples factores que intervienen y sus implicancias en los procesos de perdón y reparación, tanto en Argentina (Bombelli, Muratori, Mele, \& Zubieta, 2014) como en Chile, Paraguay y Perú (Cárdenas, Zubieta, Páez, Arnoso, \& Espinosa, 2016). Finalmente, se introdujo la dimensión de la emocionalidad colectiva a través del constructo de clima social emocional, para indagar su relación con el afrontamiento colectivo de la Justicia Transicional en estos diferentes países de sur latinoamericano (Arnoso, Páez, Cárdenas, Zubieta, Espinosa, \& Bilbao, 2015; Bobowik, Páez, Arnoso, Cárdenas, Rimé, Zubieta, \& Muratori, 2017). El estudio aquí presentado se inscribe en esta línea: indagar en la incidencia de las medidas de la justicia retributiva y el compartir social en el clima socioemocional de una sociedad con un pasado violento.

En este marco, los estudios aportan herramientas de relevancia para que en futuras investigaciones se analicen estas aristas o dimensiones en otros fenómenos de violencia y trauma social que amenacen la destrucción de la identidad grupal. El afrontamiento colectivo del trauma-como las comisiones de verdad y las disculpas públicas- son intentos por preservar o restaurar la identidad dañada, orientados a incrementar la cohesión y reconciliación intragrupal. Son herramientas que ayudan a consolidar normas e inducen 
reacciones emocionales y morales intensas en los individuos y en la sociedad toda.

\section{REFERENCIAS}

Andrieu, K. (2010). Transitional justice: A new discipline in Human Rights. Online Encyclopedia of Mass Violence. Recuperado el 4 de febrero de 2013 de http://www.massviolence.org/ PdfVersion?id_article=539. https://doi.org/10.1093/ijtj/ijp026

Arnoso, M., Cárdenas, M., Muratori, M., Zubieta, E., Páez, D., \& Bombelli, J. (2014). Argentina: Diferencias intergeneracionales ante las medidas de justicia transicional. Revista Latinoamericana de Derechos Humanos, 25(2), 151-167.

Arnoso, M., Cárdenas, M., \& Páez, D. (2012). Diferencias intergeneracionales en la mirada hacia el pasado represivo chileno. Psicología Politica, 45, 7-26.

Arnoso, M., Cárdenas, M., Páez, D., \& Beristain, C. M. (2014). Paraguay: de las violaciones a los derechos humanos a la justicia transicional. Salud \& Sociedad, 5(1), 98-114. https://doi.org/10.22199/S07187475.2014.0001.00006

Arnoso, M., Muratori, M., Páez, D., Zubieta, E., \& Cárdenas, M. (2014). La Comisión de Verdad y Justicia: percepción de eficacia en Paraguay. Revista Internacional de Investigación en Ciencias Sociales, 10(2), 154-168.

Arnoso, M., Muratori, M., Zubieta, E., Cárdenas, M., Bombelli, J., \& Páez, D. (2017). Evaluación de medidas retributivas y restaurativas luego de pasados políticos traumáticos: una mirada al caso argentino. Cuadernos FH y CS, Facultad de Humanidades y Ciencias Sociales, Universidad Nacional de Jujuy.

Arnoso, M., Páez, D., Cárdenas, M., Zubieta, E., Espinosa, A., \& Bilbao, M. (2015). Representaciones Sociales del Pasado y rituales de justicia transicional en América Latina. Cuadernos de Pesquisa, 45(156), 48-70.

Avruch, K. (2010). Truth and Reconciliation Commissions: Problems in transitional justice and the reconstruction of identity. Transcultural Psychiatry, 47(1), 33-49. https://doi.org/10.1177/1363461510362043

Beristain, C. (2005). Democratización y democratización en América Latina: Un análisis regional. Papel de las Políticas de Verdad, Justicia y Reparación. En Instituto Interamericano de Derechos Humanos (Ed.), Verdad, justicia y reparación. Desafios para la democracia y la convivencia social (pp. 53-84). San José: International IDEA.

Beristain, C. M., Páez, D., Rimé, B., \& Kanyangara, P. (2010). Psychosocial effects of participation in rituals of transitional justice: A collective-level analysis and review of the literature of the effects of TRC sans trials on human rights violations in Latin America. Revista de Psicología Social, 25(1), 47-60. https://doi.org/10.1174/021347410790193450

Bilbao, M., Techio, E., Zubieta, E., Cárdenas, M., Páez, D., Díaz, D., Barrientos, J., \& Blanco, A. (2011). Bienestar Subjetivo y Psicológico-Social: El Impacto de la Violencia Colectiva. En D. Páez, C. Martin Beristain, J. L. González, N. Basabe \& J. De Rivera. (Eds.), Superando la Violencia Colectiva y Construyendo Cultura de Paz (pp. 205-245). Madrid, España: Fundamentos.

Blanco, A. \& Díaz, D. (2004). Bienestar social y trauma psicosocial: Una visión alternativa al trastorno de estrés postraumático. Clínica y Salud, 3, 227-252.

Blatz, C., Schuman, K., \& Ross, M. (2012). Government apologies for historical injustices. Political Psychology, 30(2), 219-241. https://doi.org/10.1111/j.1467-9221.2008.00689.x

Bobowik, M., Páez, D., Arnoso, M., Cárdenas, M., Rimé, B., Zubieta, E., \& Muratori, M. (2017). Institutional Apologies and Socio-emotional Climate in the South American Context. British Journal of Social Psychology, 56(3), 578-598. https://doi.org/10.1111/bjso.12200

Bombelli, J., Muratori, M., Arnoso, M., Mele, S., \& Zubieta, E. (2013). La CONADEP y el Informe Nunca Más: conocimiento, eficacia y emociones asociadas. Anuario de Investigaciones, XX(I), 197-205.

Bombelli, J., Muratori, M., Mele, S., \& Zubieta, E. (2014). Procesos de perdón y reconciliación en argentina: aportes para una mejor comprensión de los efectos de la violencia de estado. Anuario de Investigaciones, XXI, 119-126.

Calcagno, J. L. (2013). La construcción de la democracia: Raúl Alfonsín y los militares. Buenos Aires: Corregidor.

Cárdenas, M., Páez, D., Arnoso, M. y Rimé, B. (2013). Percepción del Clima Socioemocional y la Confianza Institucional en Víctimas de Violencia Política: Valoración del Impacto de la Comisión Nacional de Verdad y Reconciliación. Psykhe, 22, 2, 111-127. https://doi.org/10.7764/psykhe.22.2.572

Cárdenas, M., Páez, D. \& Rimé, B. (2013). El impacto psicosocial de los procesos transicionales en Chile: evaluación de los efectos de las comisiones nacionales de "Verdad y Reconciliación" y "Prisión Política y Tortura”. Revista de Psicología Social, 28, 145-156. https://doi.org/10.1174/021347413806196717

Cárdenas, M., Zubieta, E., Páez, D., Arnoso, M., \& Espinosa, A. (2016). Determinantes de la aprobación del trabajo realizado por las Comisiones de Verdad y Reconciliación en el cono sur: un estudio $\begin{array}{llllll}\text { comparativo. } & \text { Revista de } & \text { Psicología } & \text { Social, } & 31(3), & 1-37 .\end{array}$ https://doi.org/10.1080/02134748.2016.1190127

Cehajic, S., Brown, R., \& Castano, E. (2008). Forgive and forget? Antecedents and consequences of intergroup forgiveness in Bosnia and Herzegovina. Political Psychology, 29, 351-368. https://doi.org/10.1111/j.1467-9221.2008.00634.x

de Rivera, J. (1992). Emotional climate: social structure and emotional dynamics. International Review of Studies on Emotion, 2, 197-218. 
Fernandez, O., Mele, S., Bombelli, J. I., \& Zubieta, E. (2011). Clima social emocional, confianza en las instituciones $y$ percepción de problemas sociales. Trabajo presentado en el $3^{\circ}$ Congreso Internacional de Investigación de la Facultad de Psicología de la Universidad Nacional de La Plata, 15-17 de Noviembre de 2011 .

Finkenauer, C. \& Rimé, B. (1998). Keeping emotional memories secret: Health and subjective well-being when emotions are not shared. Journal of Health Psychology, 3(1), 37-58. https://doi.org/10.1177/135910539800300104

Frattaroli, J. (2006). Experimental disclosure and its moderators: A meta-analysis. Psychological Bulletin, 132(6), 823-865. https://doi.org/10.1037/0033-2909.132.6.823

Garcia-Mazzieri, S. (2011). Clima social emocional y criterios de bienestar psicosocial. En Memorias del II Congreso Internacional de Investigación y Práctica Profesional en Psicología (pp. 119-122). Buenos Aires, Argentina: Facultad de Psicología, Universidad de Buenos Aires.

Hayner, P. (1994). Truth Commissions - 1974 to 1994. A comparative study. Human Rights Quarterly, 16, 597-655. https: / / doi.org/10.2307/762562

Instituto Espacio para la Memoria (2013). El Terrorismo de Estado en la Argentina. Buenos Aires: Instituto Espacio para la Memoria.

Lira, E. \& Castillo, M. (1991). Psicología de la amenaza política y del miedo. Santiago de Chile: ILAS.

Martín-Baró, I. (2003). Poder, ideología y violencia. Madrid: Trotta.

Muratori, M. \& Zubieta, E. (2013). Miedo al delito y victimización como factores influyentes en la percepción del contexto social y clima emocional. Boletin de Psicología, 109, 7-18.

Páez, D., Ruiz, J. I., Gailly, O., Kornblit, A. I., Wiesenfeld, E., \& Vidal, C. M. (1996). Clima Emocional: Su Concepto y Medición mediante una investigación transcultural. Revista de Psicología Social, 12, 79-98. https://doi.org/10.1174/021347497320892045

Pennebaker, J. W., Zech, E., \& Rimé, B. (2001). Disclosing and sharing emotion: Psychological, social, and health consequences. En M. S. Stroebe, W. Stroebe, R. O. Hannson, \& H. Schut (Eds.), Handbook of bereavement research: Consequences, coping, and care (pp. 517-539). Washington: American Psychological Association. https://doi.org/10.1037/10436-022

Preacher, K. J. \& Hayes, A. F. (2008). Asymptotic and resampling strategies for assessing and comparing indirect effects in multiple mediator models. Behavior Research Methods, 40, 879-891. https: / / doi.org/ 10.3758/BRM.40.3.879

Rimé, B. (2005). Le partage social des emotions. Paris: PUF.

Robben, A. C. (2005). How traumatized societies remember: The aftermath of Argentina's dirty war. Cultural Critique, 59(1), 120-164. https://doi.org/10.1353/cul.2005.0010

Techio, E., Zubieta, E., Páez, D., De Rivera, J., Rimé, B., \& Kanyangara, P. (2011). Clima Emocional y Violencia Colectiva: El Estado de la cuestión y los instrumentos de medición. En D. Páez, C. Martin Beristain, J. L. González, N. Basabe \& J. De Rivera. (Eds.), Superando la Violencia Colectiva y Construyendo Cultura de Paz (pp. 103-148). Madrid, España: Fundamentos.

Teitel, R. G. (2003). Transitional Justice Genealogy. Harvard Human Rights Journal, 16, 69-94.

Victoriano Serrano, F. (2010). Estado, golpes de Estado y militarización en América Latina: una reflexión histórico política. Argumentos, 23(64), 175-193.

Weinman, J., Ebrecht, M., Scott, S., Walburn, J., \& Dyson, M. (2008). Enhanced wound healing after emotional disclosure intervention. British Journal of Health Psychology, 13(1), 95-102. https: / / doi.org/10.1348/135910707X251207

Wilson, R. (1995). Manufacturing legitimacy: The Truth and Reconciliation Commission and the Rule of Law. Indicator $S A, 13(1), 41-46$.

Zubieta, E., Bombelli, J., \& Muratori, M. (2015). Argentina: el impacto de la implementación de medidas de Justicia Transicional post dictadura. Revista de Psicología Politica, 15 (32), 101-118.

Zubieta, E., Delfino, G., \& Fernández, O. (2008). Clima socioemocional, confianza en las instituciones, y percepción de problemas sociales. Un estudio con estudiantes universitarios urbanos argentinos. Psykhé, 17(1), 5-16.

Recibido 08-03-2018 | Aceptado 16-04-2018

Este trabajo se encuentra bajo una Licencia Creative Commons Atribución 4.0 Internacional que permite a terceros utilizar lo publicado siempre que se dé el crédito pertinente a los autores y a Psicodebate 